\title{
Two-Dimension Chaos-Based Signature Algorithm for Multivariate Public Cryptosystem
}

\author{
Xiaoyan Sun ${ }^{1, a^{*}}$ \\ ${ }^{1}$ Guangxi Key Lab of Multi-source Information Mining \& Security, Guangxi Normal University, Guilin \\ 541004, China \\ ajgxysxy@126.com
}

Keywords: Chaos; Perturbation; Secure; Signature; System

\begin{abstract}
Cryptography plays an important role in confidentiality, privacy protection, integrity verification. The most widely used public key cryptosystem including RSA system and elliptic curve cryptography. Since quantum algorithm and quantum computer are both developed rapidly, the current public key cryptosystem and signature scheme are under threaten, including RSA and elliptic curve cryptography. In this paper, an internal perturbation based on two-dimension chaos system is proposed to improve the security of traditional multivariate public key signature system. The outputs of central map in multivariate public key cryptosystem are acted as initializers for a two dimension chao system. When two sequences are calculated in chaos system, the corresponding elements are multiplied one by one. When both of multivariate public key cryptosystem and chaos system are estimated, the outputs of the two system are added to get the final signature. Because of the nonlinearity and unpredictability of chaos system, the proposed signature system is able to resist bilinear attack and rank attack and as a consequence, the proposed signature scheme is more secure than traditional multivariate public key signature systems.
\end{abstract}

\section{Introduction}

As the rapid development of information technology, information acts as a vital strategic resources in modern society. Information security is related to national security and social stability[1][2]. RSA cryptosystem and Elliptic Curve Cryptography (ECC) are two major signature systems widely used in network applications[3][4]. The RSA public key cryptosystem is based on the complexity of big integer factorization[5] and elliptic curve cryptography is originated from discrete logarithm[6]. These two cryptosystems are secure enough under the current computer calculate ability[7]. However, quantum algorithm developed by Peter Shor from Bell laboratory is able to attack the above two systems in polynomial time[8]. As a result, the major signature systems are not secured any more.

Post-quantum cryptography is a hot topic interesting lots of researchers. Multivariate public key cryptosystem (MPKC) is one of cryptosystems who are able to resist quantum algorithm. Besides, the computational complexity of MPKC is much less than RSA and elliptic curve cryptography[9]. The most common MPKC includes MI system developed by Matsumoto and Imai in 1988[10], Hidden Field Equation (HFE) system proposed by Patarin in 1995[11], Unbalanced Oil and Vinegar (UOV) Schemes designed by Patarin in 1997[12], Tame Transformation system originated by T.T.Moh in 1999[13]. Because of the theoretical limitation, MPKC is suitable for signature rather than encryption. For instance, there are three families about Oil-Vinegar scheme: balanced Oil-Vinegar, unbalanced Oil-Vinegar and Rainbow. Balanced Oil-Vinegar and unbalanced Oil-Vinegar are single layer scheme. While rainbow is a multilayer signature scheme. Though the Rainbow scheme was a very efficient public key signature scheme with a very high security level, it was broken in the next few years. A multivariate signature scheme SFlash was accepted by NESSIE(New European Schemes for Signatures, Integrity and Encryption) for European standard of low energy consumption intelligent card[11]. However, Sflash was broken by Dubios three years late[12]. Trap-door modification is an efficient and feasible method to improve the security of MPKC[13][14]. HFE, for example, is a modification of MI scheme with minus trap-door (HFE-) and plus-minus trap-door (HFE+-). And 
more attention is paid on other trap-door about HFE such as HFEv and HFEv_ (HFEv-minus)[16]. These modifications are inspired by Oil-Vinegar and the Minus method. There is a famous signature scheme based on Tame Transformation system as well: Tame Transformation Signature (TTS) scheme proposed by Chen and Yang in 2003. The basic idea is to apply minus trap-door on tame transformation system[17]. This paper proposes a novel internal trap-door modification in MPCK for signature. Based on the nonlinearity of chao system, a two-dimension chao system is utilized as a trap-door modification for MPKC.

\section{Basic Theory}

Internal Perturbation of MPKC. The mathematical structure of MPKC is shown in Eq. (1)[18].

$$
\left\{\begin{array}{c}
y_{1}=\sum_{1 \leq j \leq k \leq n} c_{1 j k} x_{j} x_{k}+\sum_{1 \leq j \leq n} b_{1 j} x_{j}+a_{1} \\
y_{2}=\sum_{1 \leq j \leq k \leq n} c_{2 j k} x_{j} x_{k}+\sum_{1 \leq j \leq n} b_{2 j} x_{j}+a_{2} \\
\vdots \\
y_{m}=\sum_{1 \leq j \leq k \leq n} c_{m j k} x_{j} x_{k}+\sum_{1 \leq j \leq n} b_{m j} x_{j}+a_{m}
\end{array}\right.
$$

where $a_{i}, b_{i j}, c_{i j k} \in F_{q} . q$ is a prime number and $F_{q}^{k}$ is $k$-dimension vector space on finite domain $F_{q}$ Non-linear central map $P$ is the core of Multivariate Public Key Cryptology System. The main function of $T$ and $S$ is to hide central map. Unfortunately, all basic MPKCs are proven to be insecure. However, there are some modifications called internal perturbation which can improve the security of basic MPKCs.

(1) Plus method: "+"

Plus method was first discussed by Patarin in 1996. The legitimate users insert $k$ random quadratic equations $\left(l_{1}, \ldots, l_{k}\right)$ to the central equations[19]. Let $p$ be the initial central map, then the new central map $p^{\prime}$ is derived by equation(2).

$$
\left\{\begin{array}{c}
p_{1}^{\prime}\left(x_{1}, \ldots, x_{n}\right)=p_{1}\left(x_{1}, \ldots, x_{n}\right) \\
p_{2}^{\prime}\left(x_{1}, \ldots, x_{n}\right)=p_{2}\left(x_{1}, \ldots, x_{n}\right) \\
\vdots \\
p_{m}^{\prime}\left(x_{1}, \ldots, x_{n}\right)=p_{m}\left(x_{1}, \ldots, x_{n}\right) \\
p^{\prime}{ }_{m+1}\left(x_{1}, \ldots, x_{n}\right)=l_{1}\left(x_{1}, \ldots, x_{n}\right) \\
\vdots \\
p^{\prime}{ }_{m+k}\left(x_{1}, \ldots, x_{n}\right)=l_{k}\left(x_{1}, \ldots, x_{n}\right)
\end{array}\right.
$$

(2) Minus method: “_”"

The minus method removes equations from central map rather than adds them[20]. We can see the central map from equation(3).

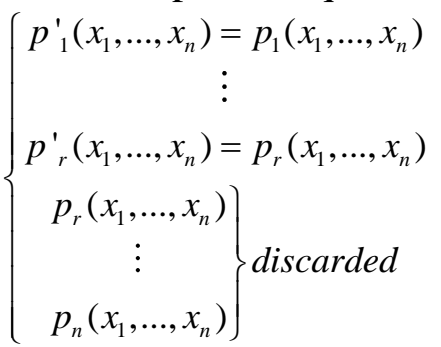

The plus method is believed to be slightly more secure than basic MPKCs. Furthermore, the minus method enhances the security of basic multivariate schemes obviously. However, most of these perturbations can only be used for signing messages.

Chaos Theory. Chaos is defined as random behavior of deterministic system. There are at least three characteristics in chaos system: being sensitive to initial values, having random-like property 
and unpredictability [21]. According to the number of variables in an equation, chaos equations can be categorized into one-dimension, two-dimension and three-dimension equations which are shown in Eq. (4), (5), (6) respectively[22].

$$
\begin{aligned}
& x_{n+1}=\lambda x_{n}\left(1-x_{n}\right) \\
& \left\{\begin{array}{l}
x_{n+1}=1+y_{n}-C_{n}^{2} \\
y_{n+1}=B x_{n}
\end{array}\right. \\
& \left\{\begin{array}{l}
\frac{d x}{d t}=\sigma(y-x) \\
\frac{d y}{d t}=\rho x-y-x z \\
\frac{d z}{d t}=x y-\beta z
\end{array}\right.
\end{aligned}
$$

Where $(x, y, z)$ represent some meaningful variables and act as system trajectory. And also system parameters $\delta, \rho, \beta$ are participating in calculation.

\section{Multivariate-Chaos Signature System}

The proposed signature system is depicted in Fig. 1. The internal trap-door modification is that the initial values are generated from the outputs of central map. In the two-dimension chaos system, a modular arithmetic is applied since the element 0 and element 1 in $F_{q}$ are both existed whatever the domain $F_{q}$ is.

Taking the first two outputs of central map as initial values of two-dimension chaos system, a novel multivariate signature system with chaos-perturbation is originated. The public key, i.e. polynomials of degree 2 over finite fields, is shown as Eq. (8).

$$
\begin{aligned}
& Y=\left(y_{1}, \ldots, y_{m}\right)=T \text { o } P \text { o } S(X) \\
& =\left\{\begin{array}{c}
y_{1}=\sum_{j, k=1}^{n} \gamma_{1, j, k} x_{j} x_{k}+\sum_{j=1}^{n} \beta_{1, j} x_{j}+\alpha_{1} \\
y_{2}=\sum_{j, k=1}^{n} \gamma_{2, j, k} x_{j} x_{k}+\sum_{j=1}^{n} \beta_{2, j} x_{j}+\alpha_{2} \\
\ldots \ldots \\
y_{m}=\sum_{j, k=1}^{n} \gamma_{m, j, k} x_{j} x_{k}+\sum_{j=1}^{n} \beta_{m, j} x_{j}+\alpha_{m}
\end{array}\right.
\end{aligned}
$$

And the private key consists of maps $S, T, p$ and $z$.

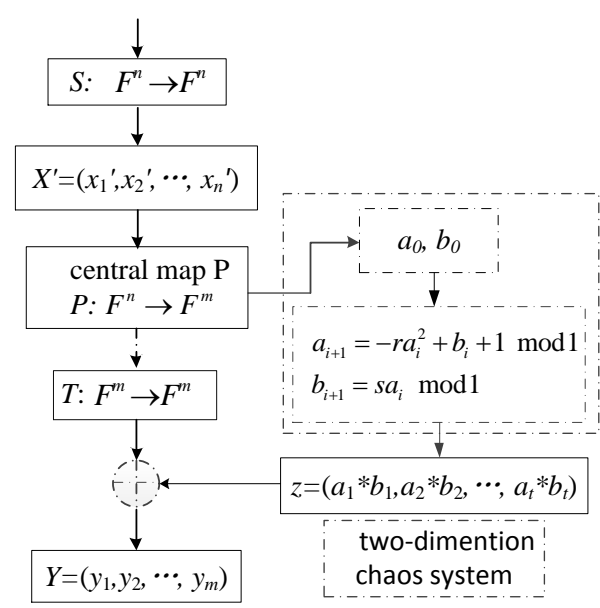

Figure 1. Diagram of Multivariate Branch Chaos Signature System 
To sign a document, which is an element $\mathrm{Y}=\left(y_{1}, \ldots, y_{m}\right)$, we need to solve the equation

$Y=F(X)=T$ o $P$ ○ $S(x)$

We must apply the inverse of $S, P$ and $T$. First, we have $Y^{\prime \prime}$ :

$Y^{\prime}=T^{-1}(Y)=P \circ S\left(x_{1}, \ldots, x_{n}\right)$

Next, we need to calculate the inversion of central map $P$. In this case, we must solve the equation

$P\left(x_{1}, \ldots, x_{n}\right)=Y^{\prime}$

It should be pointed out that the central map $P$ is not an invertible map. To overcome this problem, we can calculate the parity-check of $\left(y_{1}^{\prime}, \ldots, y_{m}^{\prime}\right)$, which can be presented as $\left(o_{1}, \ldots, o_{n-m}\right)$, and plug them into $Y^{\prime}$. As a result, the new equation is shown in Eq. (19).

$P\left(x_{1}, \ldots, x_{n}\right)=\bar{Y}=\left(\bar{y}_{1}, \ldots, \bar{y}_{n}\right)$
where $\left\{\begin{array}{l}\bar{y}_{i}=y_{i}^{\prime}, i=1, \ldots, m \\ \bar{y}_{i}=o_{i-m}, i=m+1, \ldots, n\end{array}\right.$

Thus, we can solve these invertible equations shown above and have all values of $\left(x_{1}^{\prime}, \ldots, x_{n}^{\prime}\right)$. Then we apply the inverse of $\mathrm{S}$ and estimate $X=\left(x_{1}, \ldots, x_{n}\right)=T^{-1}\left(X^{\prime}\right)$. In the next step, we will calculate chaos sequence. By taking $y_{1}^{\prime}, y_{2}^{\prime}$ as initial stimulus and specify parameters $\mathrm{B}$ and $\mathrm{C}$, we can generate chaos sequence, which we denote by $z=\left(z_{1}, z_{2}, \ldots, z_{n}\right)$. We add the plain message $Y$ and $z$ and a new value $\overline{\bar{Y}}$ is calculated.

$\overline{\bar{Y}}=Y \oplus z$

Again, taking $\overline{\bar{Y}}$ as plain messages and applying the inverse of affine transformation $T$, central map $p$ and affine transformation $S$, we obtain a totally different values $X=\left(x_{1}, \ldots, x_{n}\right)$. Finally, the signature $s=$ $\left(s_{1}, \ldots, s_{2 n}\right)$ is derived.

$s=X \| z$

To verify the signature, one needs to decompose $s$ to $\mathrm{X}$ and $z$ first. And then, one must calculate

$\overline{\bar{Y}}=F(X)$

Finally, one checks if indeed

$Y=\overline{\bar{Y}} \oplus z$

\section{Conclusions.}

A novel multivariate public key signature system is proposed in this paper. The advantages of chaos system are the nonlinearity, unpredictability and long periodicity. Taking advantage of these performances, an internal perturbation of multivariate public key signature scheme is developed. The outputs of multivariate public key cryptosystem are nonlinearly combined with the outputs of chaos system and thus, the signature is perturbed by a chaos system. Due to the nonlinearity of chaos system, the matrix relationship between plaintexts and signature is not existed and thus the bilinear attack and rank attack are both under resistance.

\section{Acknowledgment}

This work is Supported by Nature Science Foundation of Guangxi Province (No.2014GXNSFBA118268,

2014GXNSFBA118010), Project of Guangxi Key Lab of Multi-source Information Mining \& Security(No.MIMS13-06), Key project of Yulin normal university(No.2013YJZD04)

\section{References}

[1] W. Diffie, M. Hellman, "New directions in cryptography", Information Theory, IEEE Transactions on, vol. 22, no. 6, 1976, pp.644-654.

[2] S. CX, Z. HG, F. DG, C. ZF, H. JW, "REVIEW ON INFORMATION SECURITY", SCIENCE CHINA, vol. 37, no. 2, 2007,pp.129-150. 
[3] Gupta, Kamlesh, Silakari, Sanjay. ECC over RSA for Asymmetric Encryption: A review. International Journal of Computer Science Issues,vol.8, no.3-2, 2012, pp. 370-375.

[4] T. ElGamal, "A public key cryptosystem and a signature scheme based on discrete logarithms", IEEE Transactions on Information Theory - TIT, vol. 31, no. 4, 1985,pp.469-472.

[5] N. Koblitz, "Elliptic curve cryptosystems", Mathematics of computation, vol. 48, no. 177, 1987,pp.203-209.

[6] P. W. Shor, "Algorithms for quantum computation: discrete logarithms and factoring", Foundations of Computer Science, 1994 Proceedings., 35th Annual Symposium on, 1994, pp.124-134.

[7] W. W. Cao, L. Hu, Cryptanalysis of a Multivariate Public Key Encryption Scheme with Internal Perturbation Structure, Berlin: Springer-Verlag, 2009.

[8] H. Z. Wang, H. G. Zhang, Z. Y. Wang, M. Tang, "Extended multivariate public key cryptosystems with secure encryption function", Science China-Information Sciences, vol. 54, no. 6, 2011,pp.1161-1171.

[9] Y. Hashimoto, T. Takagi, K. Sakurai, General Fault Attacks on Multivariate Public Key Cryptosystems, Berlin: Springer-Verlag, 2011.

[10] T. Matsumoto, H. Imai, "Public quadratic polynomial-tuples for efficient signature-verification and message-encryption", In Advances Cryptology -EUROCRYPT, 1988, pp.419-453.

[11]J. Patarin, "Hidden Fields Equations (HFE) and Isomorphisms of Polynomials (IP): Two New Families of Asymmetric Algorithms", Advances in Cryptology-EUROCRYPT '96, 1996, pp.33-48.

[12] A. Kipnis, J. Patarin, L. Goubin, "Unbalanced Oil and Vinegar signature schemes", Theory and Application of Cryptographic Techniques-EUROCRYPT'99, 1999, pp.206-222.

[13] J. Ding, J. E. Gower, D. Schmidt, Multivariate public key cryptosystems, New York: Springer, 2006.

[14] J. Patarin, N. Courtois, L. Goubin, "FLASH, a Fast Multivariate Signature Algorithm Topics in Cryptology_CT-RSA 2001", Cryptographers' Track at RSA, 2001, pp.298-307.

[15] H. Z. Wang, H. G. Zhang, H. M. Guan, H. Q. Han, "A new perturbation algorithm and enhancing security of SFLASH signature scheme", Science China-Information Sciences, vol. 53, no. 4, 2010, pp.760-768.

[16] V. Dubois, P. A. Fouque, A. Shamir, J. Stern, "Practical cryptanalysis of SFLASH", International Crytology Conference-CRYPTO 2007, 2007, pp.1-12.

[17]X. Y. Nie, Z. H. Xu, L. Lu, Y. J. Liao, Security Analysis of an Improved MFE Public Key Cryptosystem, Berlin: Springer-Verlag, 2011.

[18] J. C. Faugere, L. Perret, High Order Derivatives and Decomposition of Multivariate Polynomials, New York: Assoc Computing Machinery, 2009.

[19]D. Smith-Tone, On the Differential Security of Multivariate Public Key Cryptosystems, Berlin: Springer, 2011.

[20]C. Wolf, Multivariate quadratic polynomials in public key cryptography, Mierlo: Leuven, 2005.

[21] S.S. Qiu, Y.F. Chen, M. Wu, Z. Ma, "Discussion on Chaotic Secure Communication and New Schemes of Chaotic Encryption", Journal of South China University of Technology(Natural Science Edition), vol. 30, no. 11, 2002, pp.75-80. 
[22] Y. Jing, G.J. shall, S.B. Yu, "An Improved Approach of Logistic Chaotic Series Encryption", Journal of Automatic Technology and Application, vol. 23, no. 2, 2004, pp.58-61. 